\title{
Fully coupled high-resolution medium-range forecasts: evaluation of the hydrometeorological impact in an ensemble framework
}

\author{
Luca Furnari $^{1}$, Linus Magnusson ${ }^{2}$, Giuseppe Mendicino ${ }^{3}$, and Alfonso Senatore ${ }^{1}$ \\ ${ }^{1}$ University of Calabria \\ ${ }^{2} \mathrm{ECMWF}$ \\ ${ }^{3}$ Universita della Calabria
}

February 8, 2021

\begin{abstract}
Fully coupled atmospheric-hydrological models allow a more realistic representation of the land surface-boundary layer continuum, representing both high-resolution land-surface/subsurface water lateral redistribution and the related feedback towards the atmosphere. This study evaluates the potential contribution of the fully coupled approach in extended-range mesoscale hydrometeorological ensemble forecasts. Previous studies have shown, for deterministic simulations, that the effect of fully coupling for short-range forecasts is minor compared to other sources of uncertainty, however, it becomes not negligible when increasing the forecast period. Through a proof-of-concept consisting of an ensemble (50 members from the ECMWF Ensemble Prediction System) seven-days-in-advance forecast of a high impact event affecting the Calabrian peninsula (southern Italy, Mediterranean basin) on November 2019, the paper elucidates the extent to which the improved representation of the terrestrial water lateral transport in the Weather Research and Forecasting (WRF) - Hydro modeling system affects the ensemble water balance, focusing on the precipitation and the hydrological response, in terms of both soil moisture dynamics and streamflow in 14 catchments spanning over $42 \%$ of the region. The fully coupled approach caused an increase of surface soil moisture and latent heat flux from land in the days preceding the event, partially affecting the lower Planetary Boundary Layer. However, when shoreward moisture transport from surrounding sea rapidly increased becoming the dominant process, only a weak signature of soil moisture contribution could be detected, resulting in only slightly higher precipitation forecast and not clear variation trend of peak flow, even though the latter variable increased up to $10 \%$ in some catchments. Overall, this study highlighted a remarkable performance of the medium-range ensemble forecasts, suggesting a profitable use of the fully coupled approach for forecasting purposes in circumstances in which soil moisture dynamics is more relevant and needs to be better addressed.
\end{abstract}

\section{Hosted file}

HYP_2021_Furnari_et_al.pdf available at https://authorea.com/users/394362/articles/ 507775-fully-coupled-high-resolution-medium-range-forecasts-evaluation-of-thehydrometeorological-impact-in-an-ensemble-framework 

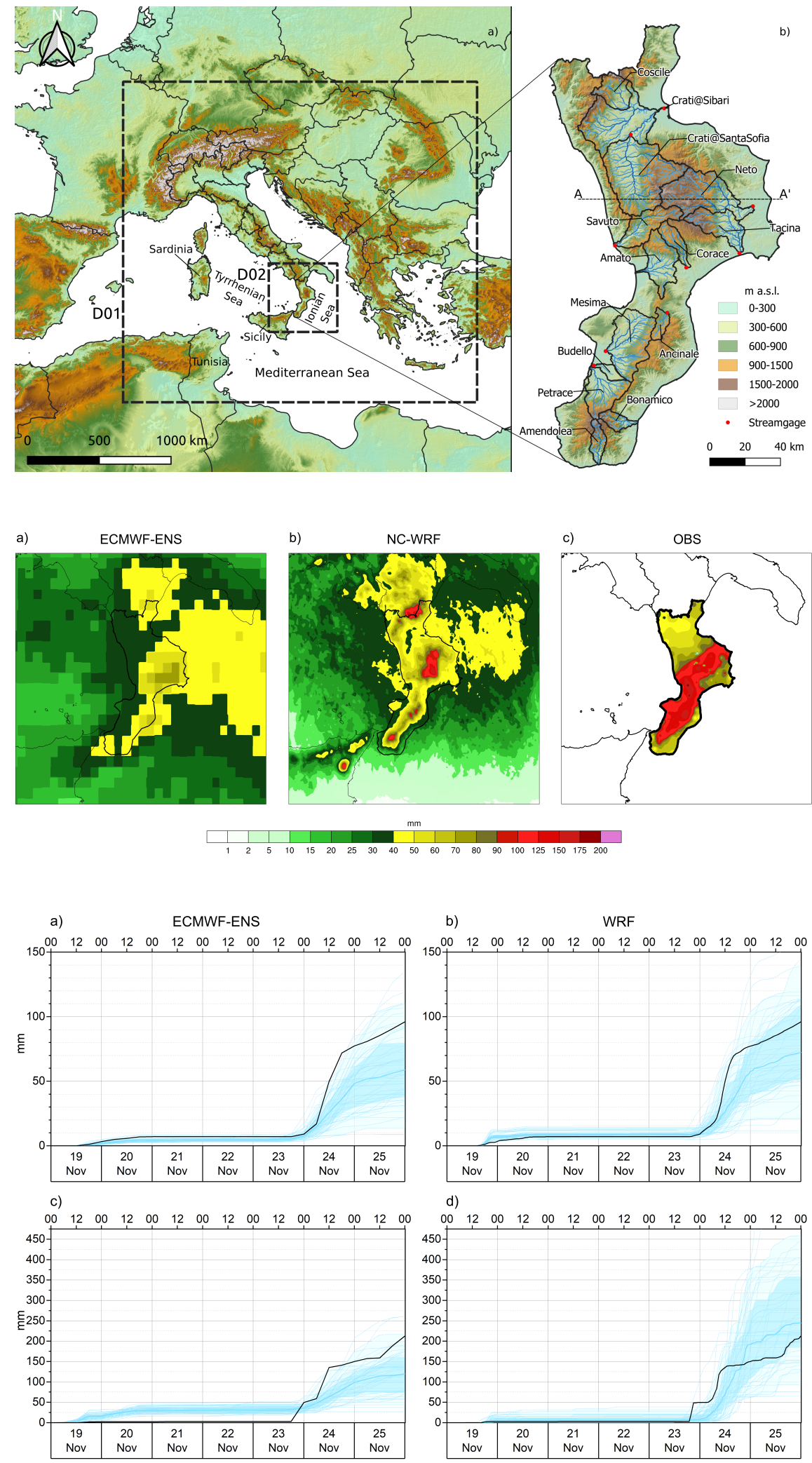

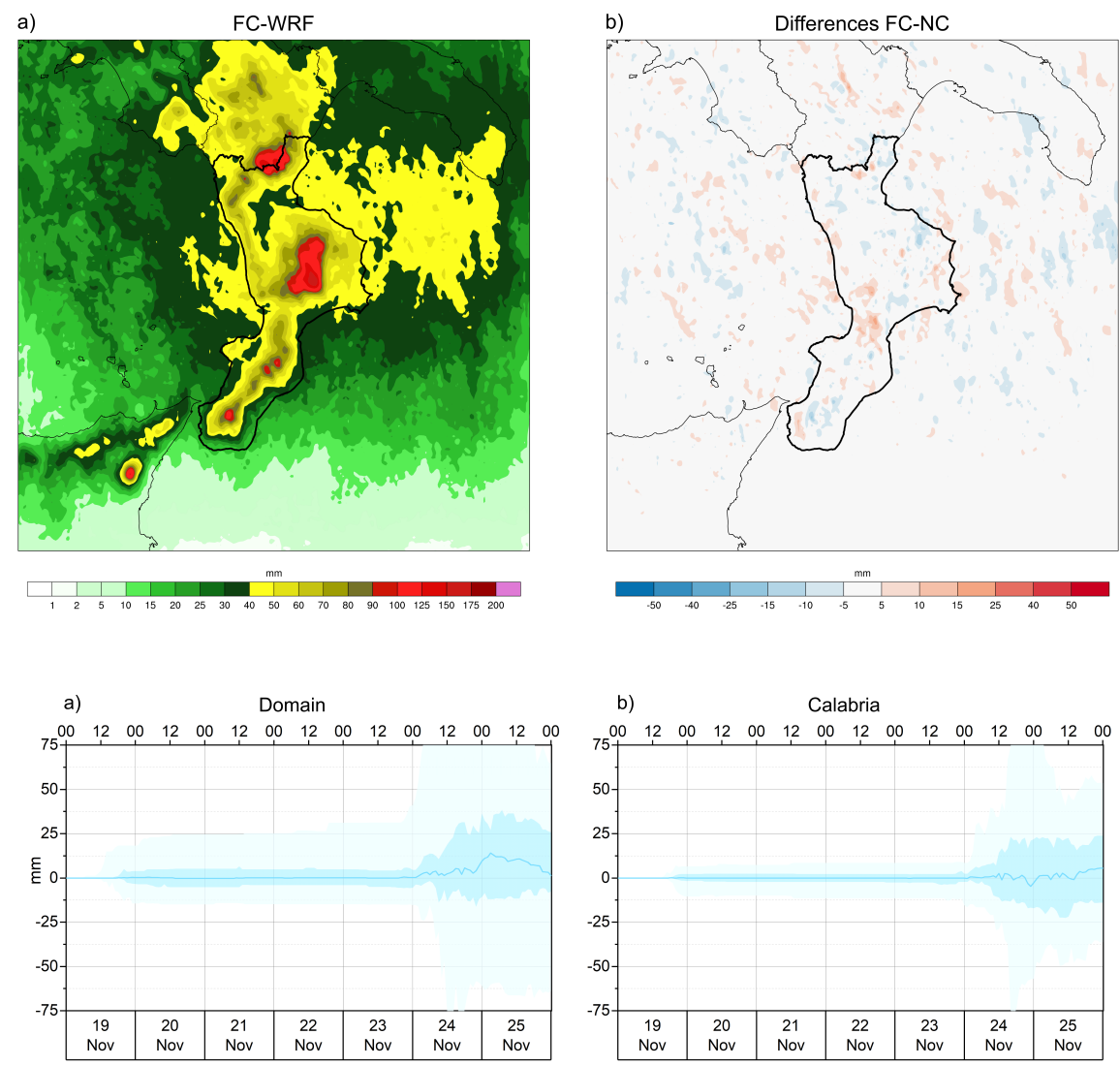

b)

Calabria

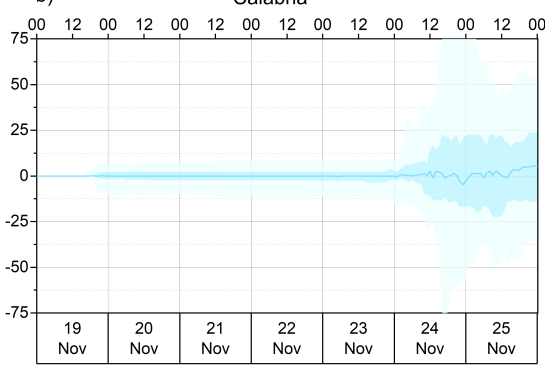




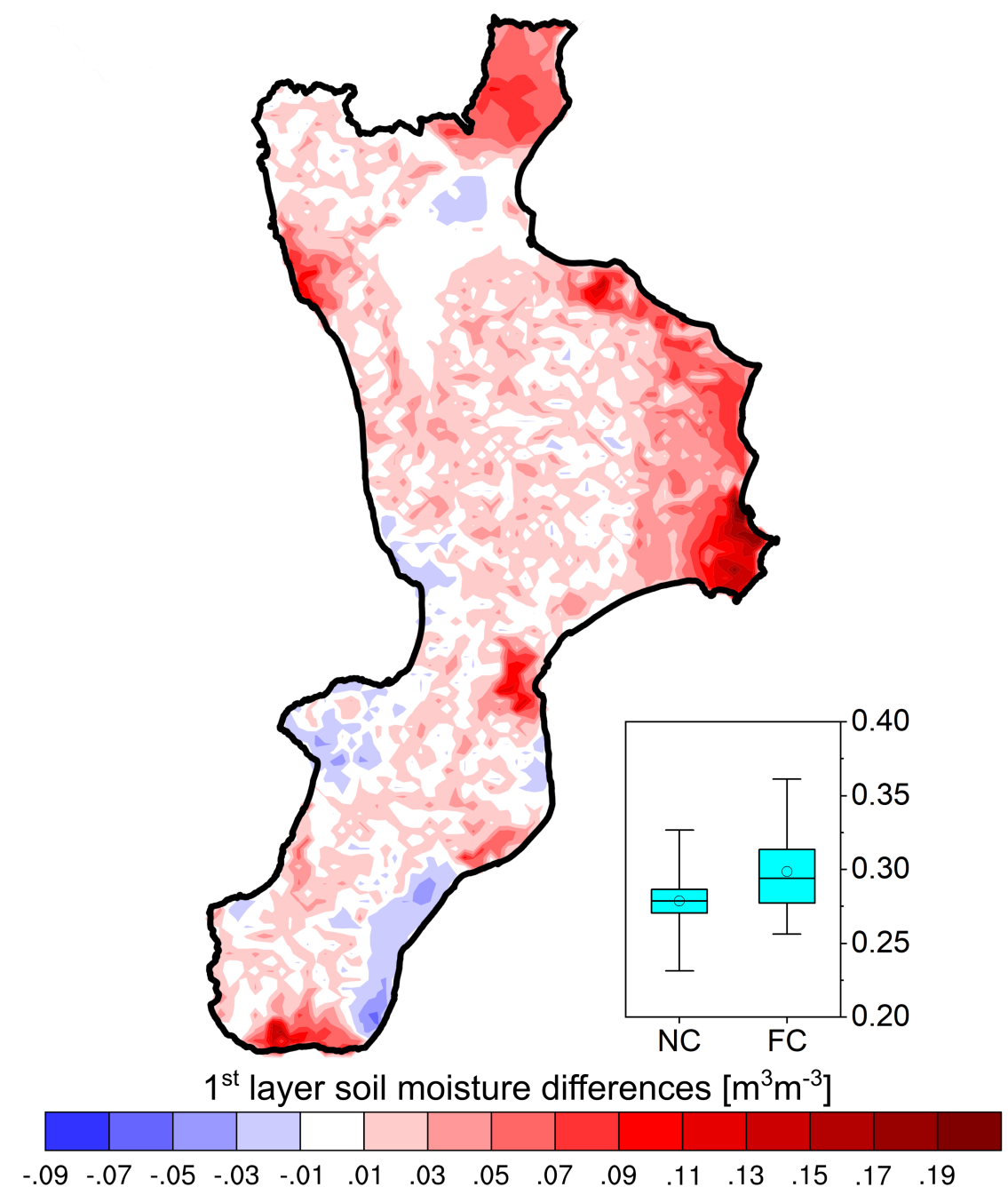



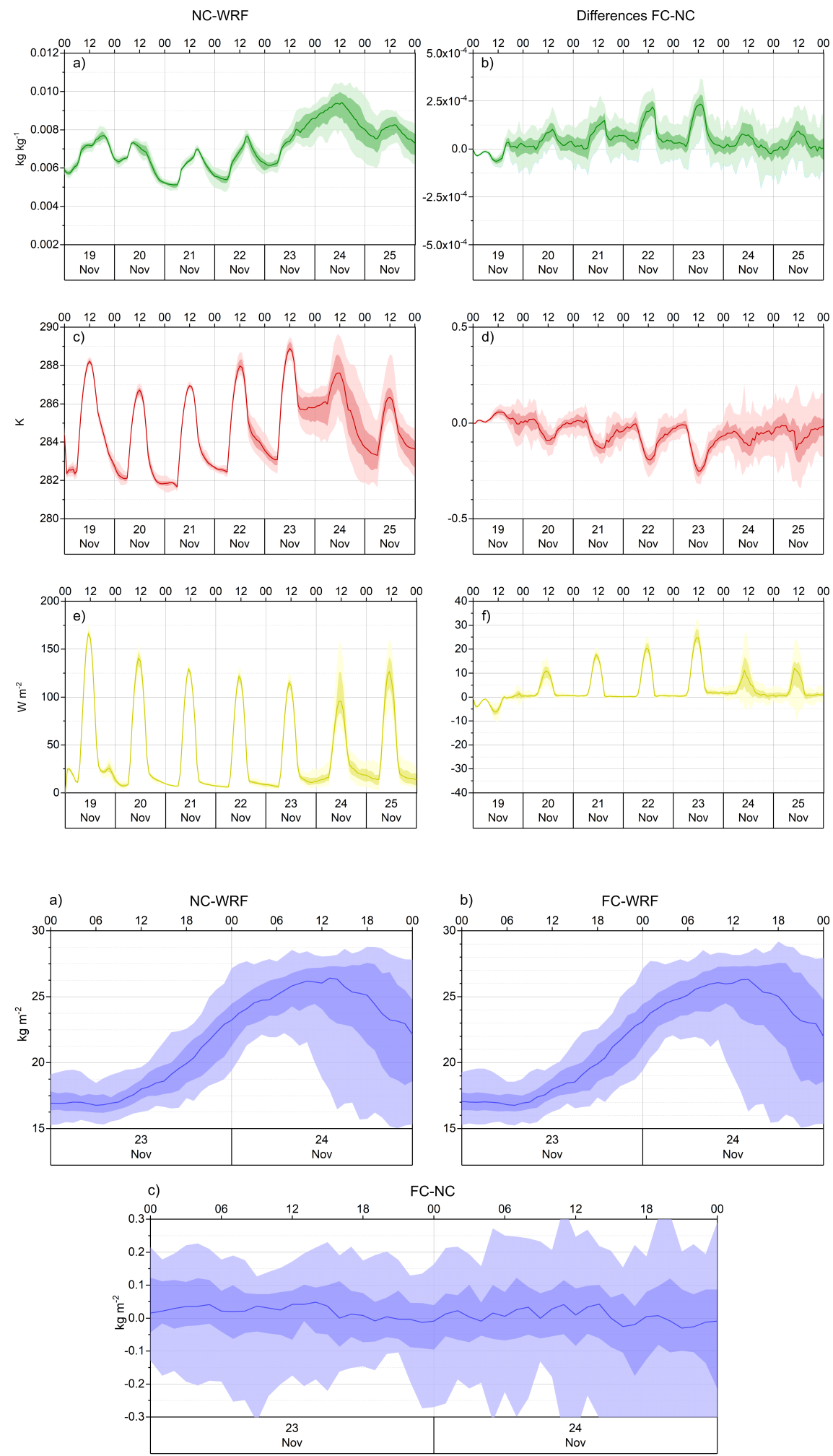

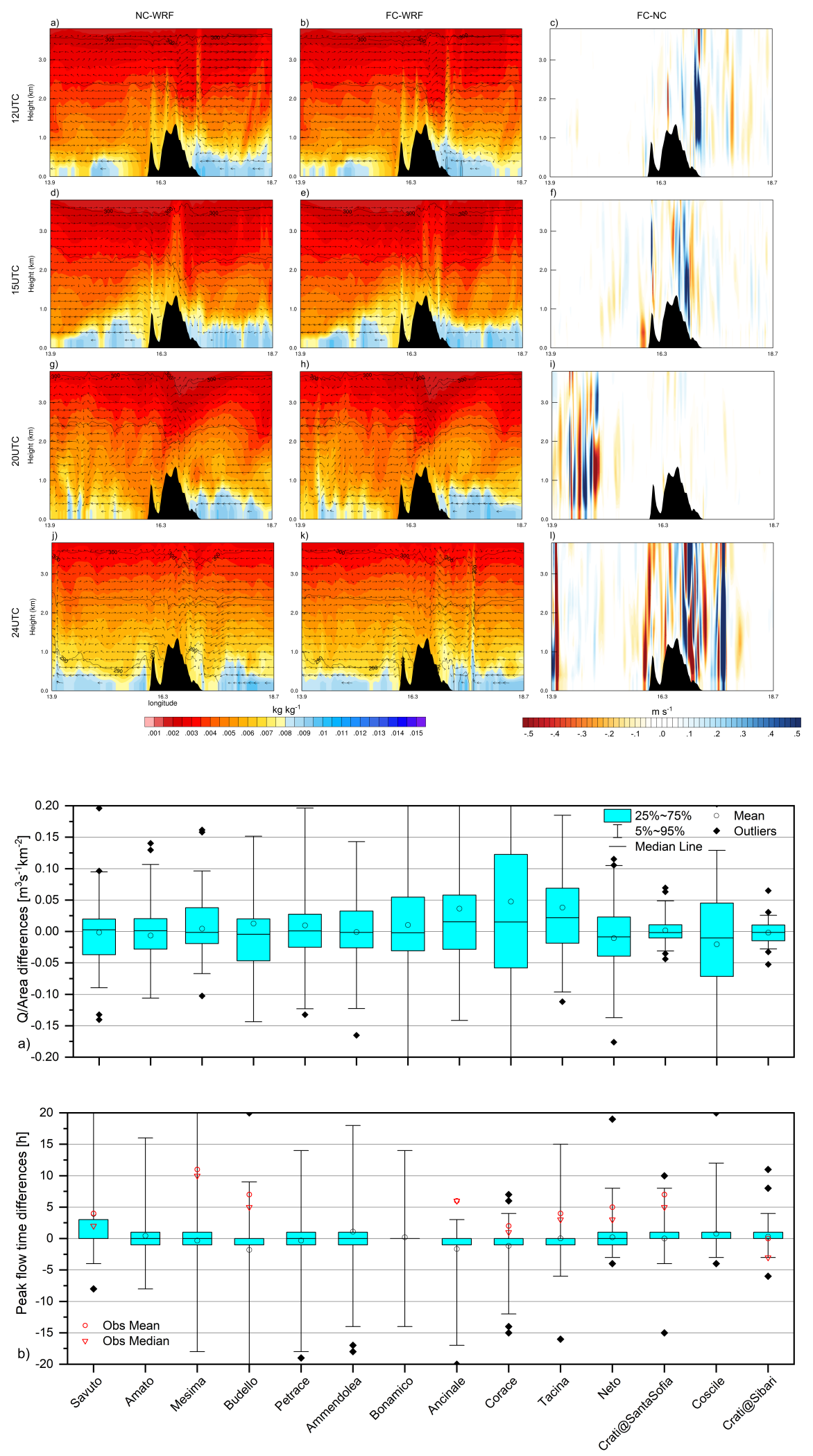